\begin{tabular}{|c|c|}
\hline Balkanologie & Balkanologie \\
\hline & Vol. $15 n^{\circ} \mathbf{2} \mid \mathbf{2 0 2 0}$ \\
\hline
\end{tabular}

\title{
Introduction. Pour une approche socio-historique de l'action collective dans les Balkans
}

Introduction. For a socio-historical approach to collective action in the Balkans

\section{Sarah Sajn et Catherine Samary}

\section{(2) OpenEdition}

\section{Journals}

Édition électronique

URL : http://journals.openedition.org/balkanologie/2573

DOI : $10.4000 /$ balkanologie. 2573

ISSN : 1965-0582

Éditeur

Association française d'études sur les Balkans (Afebalk)

Référence électronique

Sarah Sajn et Catherine Samary, «Introduction. Pour une approche socio-historique de l'action collective dans les Balkans », Balkanologie [En ligne], Vol. $15 n^{\circ} 2$ | 2020, mis en ligne le 01 décembre 2020, consulté le 23 avril 2021. URL : http://journals.openedition.org/balkanologie/2573 ; DOI : https:// doi.org/10.4000/balkanologie.2573

Ce document a été généré automatiquement le 23 avril 2021.

(c) Tous droits réservés 


\title{
Introduction. Pour une approche socio-historique de l'action collective dans les Balkans
}

\author{
Introduction. For a socio-historical approach to collective action in the Balkans
}

\author{
Sarah Sajn et Catherine Samary
}

1 Quasi disparus des radars médiatiques occidentaux, suite aux guerres yougoslaves, les Balkans se sont réinvités dans l'actualité européenne des années 2010 à la faveur de mouvements de protestation de masse : mobilisations contre les politiques d'austérité en Grèce, « bébolution » et plenums en Bosnie-Herzégovine, révolution des couleurs en Macédoine, manifestations contre la corruption en Roumanie, Bulgarie, comme dans l'espace post-yougoslave et, dans une moindre mesure, lutte pour la protection d'espaces publics dans différentes localités. De nombreux observateurs y ont vu l'écho, dans la péninsule, d'un mouvement global de protestation, initié par les «printemps arabes" et d'autres mouvements en Europe et en Amérique (Indignés en Espagne, Occupy Wall Street aux États-Unis, Gezi Park en Turquie, Maïdan en Ukraine, Gilets jaunes en France), visant principalement les logiques de (re)distribution des ressources ${ }^{1}$.

Dans le même temps, pourtant, la problématique migratoire de la « route des Balkans ", la recrudescence de mouvements nationalistes, comme des enjeux géostratégiques, ont réactivé une vision particulariste du Sud-Est européen ${ }^{2}$. Les discours médiatique, politique et même scientifique ont en effet rappelé à quel point la compréhension des Balkans reste marquée par les stigmates d'une représentation façonnée au début du $\mathrm{xx}^{\mathrm{e}}$ siècle. Médias et experts ont remis au goût du jour le terme de " poudrière ", brandi la menace de nouveaux conflits et appelé à une intervention accrue des pouvoirs occidentaux ${ }^{3}$. Ces approches sécuritaires ont réaffirmé l'assignation des Balkans au nationalisme, aux conflits ethniques et/ou religieux et à la violence, mais aussi au clientélisme et à la corruption. Malgré la multiplication des causes et des formes des mobilisations collectives, les citoyens et citoyennes de la région demeurent perçus au prisme d'une certaine soumission aux diktats de leurs communautés, de leurs 
représentants ou de puissances étrangères. À ce titre, la littérature académique en science politique sur les Balkans, continue d'être dominée par des approches surplombantes, aux tendances « groupistes ${ }^{4}$.

3 L'objectif de ce numéro est double. Premièrement, il entend contribuer à lever le voile de plomb qui pèse encore - a fortiori dans l'espace francophone - sur la connaissance des mobilisations collectives qui nourrissent la vie politique dans le Sud-Est européen. Il s'agit d'appréhender « par le bas » les dynamiques sociales, politiques et économiques contemporaines dans les Balkans. Deuxièmement, ce numéro a pour objectif de resituer ces mobilisations collectives dans leurs historicités locale, régionale et globale. Considérant que "l'espace est l'héritier du temps " ${ }^{5}$, les cinq contributions rassemblées ici invitent à penser les déterminants politiques et historiques de l'action collective contemporaine. Une attention particulière sera portée à la reconfiguration des clivages et des héritages dans le contexte spécifique des années dites de transition. Les articles s'inscrivent dans la continuité de recherches récentes questionnant les dogmes libéraux au prisme desquels la région demeure appréhendée depuis une trentaine d'années ${ }^{6}$.

\section{La remise en cause de la transition libérale}

4 Aux représentations essentialistes plus anciennes des pays de la région, la lecture dominante des bouleversements majeurs des années 1989-1991 a en effet ajouté son lot de stéréotypes. Ceux-ci ont influencé tant les politiques mises en œuvre que les paradigmes de la recherche académique. Dans le contexte dit de transition postcommuniste, les sociétés balkaniques - comme l'ensemble du «bloc de l'Est »- ont été taxées d'immaturité démocratique ${ }^{7}$. La chute du mur de Berlin est allée de concert avec la représentation de l'Europe de l'Est comme une région isolée, devant rattraper son retard dans la marche vers la globalisation capitaliste. Cette lecture implique que les citoyens étaient pris en otage par les systèmes socialistes ${ }^{8}$. Le discours du "retour à l'Europe $»^{9}$, ayant émergé de cette géographie nouvelle, a fait de la libéralisation économique et politique la pierre angulaire de la réunification du continent. Cela s'est notamment traduit par un soutien aux courants libéraux et aux « sociétés civiles » des pays dits en transition. Les réformes promues se sont donc accompagnées de financements répondant à l'impératif de création de sociétés civiles et impliquant professionnalisation et standardisation de l'action collective ${ }^{10}$. De fait, ce contexte politique a largement participé à biaiser l'écriture d'une histoire des mobilisations collectives dans les Balkans.

5 La décennie 2010 marque la progressive remise en cause du discours de rattrapage, dominant jusque-là, et l'apparition de protestations de masse. Les critiques adressées au récit de la transition et de la route vers l'Europe apparaissent aux différentes extrémités du spectre politique, des frondes nationalistes d'extrême droite aux indignés et insoumis d'extrême gauche. De plus, la remise en cause de la transition libérale consacre un bouleversement profond des clivages, des stratégies et des étiquettes politiques (populisme de gauche, démocratie illibérale...). Toutefois, des mouvements se réclamant de gauche, et tentant de réactiver l'héritage socialiste, émergent dans les Balkans. La montée de grèves massives en Grèce contre les politiques d'austérité et l'arrivée au pouvoir de la coalition de gauche radicale Syriza ont un fort impact dans le reste des Balkans ${ }^{11}$ : Alexis Tsipras est invité dans plusieurs pays, 
notamment pour la création d'un nouveau parti de gauche en Slovénie. En parallèle, le festival subversif de Zagreb, ses forums ainsi que les activités organisées par des étudiant·e's et jeunes chercheur'e's slovènes (Université Workers \& Punk, centres de recherche sociale) alimentent aussi une politisation de la crise de 2008. Les désillusions concernant le positionnement de la direction de Syriza, qui accepte de se soumettre aux exigences des instances européennes malgré le « non » majoritaire au référendum, marque l'émergence de nouveaux débats au sein de la gauche européenne ${ }^{12}$.

Dans le même temps, de nouvelles recherches se sont emparées du renouvellement des questionnements sur le passé socialiste, les crises multiples qui ont marqué la fin du " court $\mathrm{xx}^{\mathrm{e}}$ siècle ${ }^{13}$ et la place de l'Europe de l'Est dans ces bouleversements historiques. Nourris par les théories de la dépendance en termes de "système-monde ", par les approches globales de l'histoire ou les courants décoloniaux ${ }^{14}$, ces travaux ont entrepris de réinsérer, à nouveaux frais, les transformations de l'Europe de l'Est dans l'histoire récente du capitalisme ${ }^{15}$. Ces études soulignent que l'histoire de la globalisation est faite d'interconnexions multiples, de clivages politiques et idéologiques, ou encore de cycles. Ainsi, 1989 est réinterprétée, dans une perspective critique des narrations de libération nationale, au prisme du cycle néolibéral de globalisation initié par la crise des années 1970. Cette démarche met l'accent sur le démantèlement de l'État social, sans négliger les processus contemporains de création de frontières culturelles, raciales ou religieuses. Elle permet aussi de montrer comment les élites communistes ont également participé à faire de la globalisation ce qu'elle est devenue après $1989^{16}$.

7 Les recherches sur les Balkans qui, depuis quelques années, ont initié un réexamen critique de la littérature sur le socialisme et ladite transition prennent largement part à ce mouvement. En témoigne l'historiographie critique dont la Yougoslavie fait l'objet, aux antipodes du révisionnisme nationaliste triomphant au sein des partis de gouvernement. Ces recherches se fixent en effet l'objectif de lever les tabous idéologiques inhérents à la doxa de la transition. Elles réinsèrent l'analyse des classes, du genre et des différenciations sociales dans l'examen des sociétés passées et présentes ${ }^{17}$. La spécificité de l'expérience yougoslave est réévaluée au plan historique et épistémologique, notamment à la lumière d'approches marxistes hétérodoxes en termes d'« utopies concrètes $»^{18}$. Ces travaux questionnent, sous des angles inexplorés, le système autogestionnaire. Certains réintègrent l'espace yougoslave dans une histoire coloniale et raciale globale ${ }^{19}$. D'autres analysent les effets de l'expérience yougoslave à partir de recherches de terrain entreprises au cours de la dernière décennie ${ }^{20}$. D'autres encore s'intéressent aux différentes perceptions de cette expérience à l'international, comme un récent volume de la Revue d'études comparatives Est-Ouest qui, en outre (et pour la première fois dans une publication en français), donne un aperçu de la recherche historique actuelle sur la Yougoslavie ${ }^{21}$.

\section{Historiciser l'analyse de l'action collective}

8 Notre dossier s'inscrit dans la continuité de cet effort de retour critique sur les mutations des systèmes politiques, économiques et sociaux dans les Balkans, en proposant une approche socio-historique de l'action collective. Il s'agit de renouveler les analyses de science politique sur les mobilisations collectives, en s'inspirant des recherches précédemment citées, majoritairement réalisées en histoire. La publication 
d'articles en français et en anglais traduit aussi la volonté de rendre visible les points de vue et contributions de chercheurees de la région, souvent non francophones. Un certain nombre de recherches sur les mobilisations dans la péninsule balkanique ont en effet été publiées ces vingt dernières années, principalement en langue anglaise ${ }^{22}$. Reconnaître la capacité d'agir des citoyen-ne's et rendre compte du foisonnement politique, social et culturel qui caractérise les sociétés de la région est le plus grand apport de ces travaux ${ }^{23}$. Ils constituent une contribution indispensable au champ de la sociologie des mouvements sociaux et des organisations, bien qu'ils y restent largement sous-représentés ${ }^{24}$.

9 Cependant, un certain nombre de travaux sur les organisations dites citoyennes ont repris à leur compte la logique évoquée plus haut de construction des "sociétés civiles » et d'ONGisation ${ }^{25}$. La multiplication d'organisations, répondant aux critères de la démocratie libérale et du néolibéralisme, s'est donc accompagnée du développement d'une expertise scientifique sur le sujet. La notion de "société civile» a un temps envahi les revues scientifiques et les collections d'ouvrages sur la région ${ }^{26}$. Majoritairement utilisée dans le cadre d'études portant sur la démocratisation, l'européanisation ou la réconciliation ${ }^{27}$, cette notion a véhiculé toute une série de préjugés culturels et politiques dans l'approche de l'action collective et de la citoyenneté dans les Balkans. Or, la notion de société civile a largement été critiquée pour son élasticité, son caractère dépolitisant et sa problématique transposition dans des contextes étrangers à ceux dans lesquels elle a été élaborée ${ }^{28}$. Pour les tenants de cette approche, il s'agit de savoir si la société civile locale ou régionale est suffisamment développée, efficace voire mature pour contribuer à des processus politiques dont les enjeux de pouvoir ne sont que rarement discutés. La perspective évaluatrice de ces travaux a fait perdurer les idées de la «transitologie » et d'un certain retard à rattraper dans l'intégration des Balkans à la globalisation et l'européanisation ${ }^{29}$. Cette approche, qui reste influente malgré les critiques qui lui ont été adressées, a contribué à éclipser la complexité et la diversité des formes de l'action collective comme des pratiques de la citoyenneté dans la région ${ }^{30}$. Il semble pourtant indispensable de distinguer les analyses de l'action collective des critères d'évaluation édictés par les acteurs eux-mêmes si l'on entend mener un travail critique en sciences sociales.

10 Cette démarche critique nous amène aussi à reconsidérer les étiquettes et les oppositions, souvent usées, dévoyées, trop rapidement utilisées pour qualifier les mobilisations. Les termes d'européens et d'anti-européens, d'ethniques, de nationalistes ou de civiques ou encore de libéraux, de conservateurs ou de socialistes doivent être utilisés avec précaution et non pas comme catégories d'analyse. Ici encore, il s'agit de se distancier du vocabulaire des acteurs qui tendraient à légitimer ou délégitimer telles ou telles mobilisations, telles pratiques ou revendications par des qualificatifs fortement chargés symboliquement et politiquement. Dans ce dossier, nous préférons historiciser les catégories et comprendre les usages qu'en font les acteurs des mobilisations.

11 Des travaux critiques dans le champ des mouvements sociaux ont initié une déconstruction des catégories de pratiques trop souvent reprises dans les analyses ${ }^{31}$ pour tenter de les dépasser ${ }^{32}$. Ils proposent une analyse des déterminants des crises sociales, politiques et économiques dans la région, en incluant le rôle des élites locales mais aussi l'impact des politiques prônées par les organisations internationales et 
notamment européennes ${ }^{33}$. Ces travaux ont mis en avant les phénomènes contemporains de résistances discursives et pratiques aux modèles néolibéraux, néocoloniaux et nationalistes ${ }^{34}$. Certains se sont attachés à montrer la nouveauté voire la renaissance des mouvements sociaux dans la région. D’autres ont évoqué la «nouvelle gauche» balkanique ${ }^{35}$. Ces recherches ont largement interprété les mobilisations des années 2010 comme des réactions à la crise - économique, politique, sociale, écologique - et aux espoirs déçus d'une «transition » vers la démocratie et l'économie de marché. Les contributions proposées ici participent à la compréhension des liens complexes entre les mobilisations et le contexte politique, social et économique contemporain. Cependant, leur principal objectif est de mettre en lumière les reconfigurations, à travers le temps, des acteurs, des réseaux, des cadrages et des formes de l'action collective. Dans cette perspective, il s'agit de produire une analyse des contraintes et des opportunités qui ont influencé les mobilisations collectives dans les Balkans au cours de cette dernière décennie.

Nous nous intéressons donc à ce que l'histoire apporte à la compréhension des enjeux contemporains et à l'étude des mouvements sociaux, en s'inspirant de ce que Michel Offerlé posait comme acquis dans son chapitre portant sur les apports de l'histoire à l'étude des protestations :

il n'est de bonne sociologie qu'adossée fortement à un usage de l'histoire et du travail historique qui ne soit pas purement cosmétique ou instrumental (rappel chronologique, généalogie approximative, invocation de la tradition, prurit mémoriel). (...) On écrit dans son temps et pour son temps l'histoire d'un passé et c'est pourquoi la production historienne doit intéresser le sociologue, simplement féru de curiosité, de comparatisme temporel et d'interdisciplinarité. ${ }^{36}$

13 Ainsi, nous avons souhaité que les analyses des diverses formes d'action collective produites dans ce numéro intègrent autant que possible des approches sociohistoriques ou historiques du politique. La socio-histoire se construit au carrefour de l'histoire, de la sociologie et de la science politique et a, en effet, contribué à renouveler les sciences sociales en France depuis les années 1990. Elle se considère plutôt comme une «boîte à outils ${ }^{37}$ que comme une théorie unifiée, en se donnant comme objectif de «mettre en lumière l'historicité du monde dans lequel nous vivons, pour mieux comprendre comment le passé pèse sur le présent $»^{38}$. En cela, elle constitue une entrée qui nous paraît intéressante pour nourrir l'étude de formes diverses d'action collective dans une région dont l'histoire a pu être tronquée sinon occultée, comme nous l'avons mentionné plus haut.

14 Le recours à l'approche socio-historique peut également produire des travers causaux, déterministes, structuraux ou fonctionnalistes ${ }^{39}$. Cependant, François Buton rappelle que dans la socio-histoire, il y a aussi « un espace pour le décryptage des 'processus inaboutis' et des 'possibles écartés', c'est-à-dire des possibilités qui, ouvertes dans certaines conjonctures, n'ont finalement pas été concrétisées ${ }^{40}$. Une manière d'appréhender les temporalités se rapprochant de la philosophie révolutionnaire de Walter Benjamin ${ }^{41}$ qui incitait en effet à s'orienter contre les visions linéaires de l'histoire contenues dans l'idée de progrès, qu'il qualifiait de «temps homogènes et vides $»$.

15 Dans la lecture d'un mouvement social contemporain, il ne s'agit donc pas d'emprunter à une quelconque quête des origines, fussent-elle alternatives à l'histoire dominante, mais 
de donner à voir ce que ce mouvement actuel doit au passé (en termes de modalités d'action, de catégories d'analyse du politique et du monde social, etc.) et la façon dont ces héritages sont reconfigurés à l'aune de structures sociales et politiques nouvelles. L'objectif ne serait alors pas de dégager une chaîne causale conduisant irrémédiablement aux mobilisations actuelles, mais d'affirmer qu'elles ne sont compréhensibles qu'à travers l'analyse simultanée d'une histoire qui les rend possibles et d'un présent qui les voit naître. ${ }^{42}$

Les apports de l'histoire à l'étude des mouvements sociaux sont aussi essentiels que multiples. Des écrits de Thompson sur la working class anglaise ${ }^{43}$ à l'œuvre de Doug McAdam, Sidney Tarrow, Willian Sewell et Charles Tilly ${ }^{44}$ sur les contentious politics jusqu'aux plus récentes études de Gérard Noiriel ${ }^{45}$ ou Mathilde Larrère ${ }^{46}$, il semble vain d'en proposer un résumé ou un état de la littérature. Les contributions réunies dans ce numéro n'ont donc pas l'ambition d'égaler ces travaux séminaux mais de s'en inspirer, car, comme le rappelle Michel offerlé :

Un travail socio-historien, orienté vers ici l'étude des protestations, visera moins à la recherche d'une improbable exhaustivité (...) ou à l'épuisement d'un ensemble de cartons, qu'à un questionnement d'objet sur un terrain. ${ }^{47}$

\section{Les contributions}

Dans ce numéro, nous avons tenu à regrouper des contributions recouvrant différentes historicités : de la temporalité postsocialiste, notamment en Serbie et en Slovénie, couplée à celle du post-conflit en Macédoine, à l'histoire du rapport de l'armée à l'État en Grèce. Elles concernent cependant deux sous-ensembles distincts: des pays anciennement socialistes et la Grèce. Les premiers proviennent tous, pour les raisons contingentes qui sont évoquées plus bas, de l'espace post-yougoslave. Les articles en illustrent les spécificités nationales, mais chaque cas d'étude stimule des réflexions comparatives propres à enrichir la compréhension d'enjeux au sein de la région et audelà. Un éclairage de la question de la "transition", souvent rattachée aux seules situations dites postsocialistes, nous est également donné par le dernier article portant sur les luttes antimilitaristes en Grèce depuis la chute de la dictature des colonels. Se distinguant des précédentes études de cas par le contexte abordé, cette contribution permet de faire un pas de côté pour penser autrement la question des transformations politiques liées à l'adoption de la démocratie libérale et à l'intégration dans l'Union européenne. Les mobilisations étudiées dans le numéro explorent des espaces divers des usines à la rue en passant par les universités. Les articles traitent de causes variées relevant de luttes sociales, économiques, culturelles et identitaires et sont problématisés en termes de classe ou de genre. Enfin, ils incluent des formes d'actions collectives hétéroclites impliquant des syndicats, des partis, des collectifs et des associations parfois faiblement structurées, jusqu'aux protestations de masse.

Jelena Pešić et Jelisaveta Petrović s'intéressent au mouvement « Un sur cinq millions » qui a éclaté en Serbie fin 2018. Après plusieurs protestations de masse en 2016 et 2017, ce mouvement a duré un an et s'est étendu à plus de cent villes, constituant le plus important mouvement de protestation de l'ère post-Milošević. Les auteures se basent sur ce cas d'étude significatif pour examiner les déterminants du développement et du positionnement des organisations se revendiquant de gauche sur la scène politique serbe. Elles montrent que, malgré un contexte économique et politique a priori favorable à la diffusion d'une critique anticapitaliste (crise de 2008, politiques 
d'austérité, déception du post-socialisme), les organisations de gauche ne parviennent pas à imposer une lecture socioéconomique des problématiques contemporaines. Les transformations socioéconomiques ont également fragmenté les classes paupérisées et les ont éloignées des organisations de gauche, majoritairement constituées d'une élite urbaine et diplômée. En resituant le mouvement "un pour cinq millions" dans l'histoire des transformations politiques, sociales et économique du pays, les auteures analysent les conditions socio-historiques spécifiques qui ont entravé la popularité et l'impact des forces politiques de gauche. Leur interprétation est construite à travers une analyse en termes de structure d'opportunité discursive. Celle-ci est marquée par " deux décennies de stigmatisation et de dépréciation du communisme » après la chute de Slobodan Milošević en 2000, alors qu'il avait choisi de rebaptiser l'ancienne Ligue des communistes en parti «socialiste ». Le discrédit de sa politique et les conditions de son échec ont donc été sous-jacents à la «marginalisation de la gauche dans l'arène politique en Serbie » au cours des décennies suivantes. Les auteures insèrent ainsi dans une historicité spécifique leurs pistes de réflexion sur les contraintes structurales qui pèsent sur l'émergence de forces progressistes de masse dans la Serbie post-Milošević et postsocialiste.

19 La contribution de Biljana Vankovska porte sur d'autres protestations de masse dont l'épilogue est connu sous le nom de "révolution des couleurs"(colorful revolution). Celles-ci se déroulent au cœur d'une des anciennes républiques parmi les moins développées de la fédération, la Macédoine, qui s'est consolidée en tant qu'État dans la Yougoslavie titiste. L'auteure, actrice au sein de ces mobilisations, donne à voir une autre difficulté dans l'émergence d'une alternative progressiste autonome, celle de la périphérisation géopolitique. Son approche socio-historique situe ces mobilisations au sein des transformations qui ont constitué la scène politique et le jeu des partis en Macédoine au cours des vingt-cinq dernières années. Celles-ci sont de trois ordres : la dissolution yougoslave, les conflits qui ont affecté son territoire et celui des républiques voisines, ainsi que l'intervention internationale dans la région. En prenant le contre-pied d'autres analyses sur ces mobilisations, elle montre comment les tentatives d'action collective impliquant étudiants et professeurs d'université se confrontent aux routines politiques nées du processus de state-building et sont instrumentalisées par un système politique impliquant acteurs locaux et internationaux. Ces mobilisations ont connu un fort engouement médiatique et politique dépassant le cadre régional. L'analyse qu'en propose l'auteure met en lumière le fonctionnement et l'impact $\mathrm{du}$ mode de gouvernement internationalisé caractéristique de la Macédoine post-conflit. Selon Vankovska, des acteurs régionaux et internationaux ont utilisé la dimension ethnique comme une ressource politique. En effet, en étudiant la chronologie et la sociologie des mouvements sociaux se succédant sur plusieurs années, l'auteure montre comment les principes ethno-nationaux de gouvernement, souvent associés à une histoire et une spécificité régionale, sont consolidés par l'action de la communauté internationale, écartant de la sorte un mouvement social aux cadrages politiques alternatifs. À travers une analyse des mouvements sociaux de 2014-2016 ancrée dans l'histoire politique macédonienne depuis la fin de l'ère yougoslave, l'auteure expose alors les contraintes pesant sur l'engagement civique dans cette super-périphérie européenne.

20 L'article de Maja Breznik et Boris Mence porte sur la Slovénie, la plus riche des anciennes républiques yougoslaves. Il s'intéresse aux différenciations entre travailleurs analysées à partir des outils conceptuels fournis par l'opéraïsme italien, un courant qui 
distingue analytiquement la composition technique et politique de la classe ouvrière. L'article s'appuie sur une vaste enquête, initialement commanditée par un syndicat, menée dans trois entreprises du pays lors de la récession de 2013-2014. L'interprétation des différenciations entre travailleurs au sein des usines étudiées s'inscrit dans une approche socio-historique qui distingue différentes phases dans la transition libérale slovène depuis l'indépendance de 1991, au prisme de ce qui a fait sa spécificité dans toute la région: le rôle des syndicats. L'étude retrace l'évolution des stratégies syndicales sur la période, marquée par les changements de majorité politique au pouvoir et les pressions émanant des institutions européennes. Elle relève comment, grâce à l'ampleur des mobilisations syndicales, la république slovène a initialement mieux résisté que d'autres aux préceptes néolibéraux, qui se sont toutefois imposés. Afin de saisir les attentes des travailleurs vis-à-vis des syndicats dans les usines étudiées, et notamment des travailleurs plus précarisés, Maja Breznik et Boris Mence mettent ces attentes en perspective avec les évolutions des relations entre le capital et le travail, d'une part, et entre les syndicats et leurs membres, d'autre part. Les auteurs soulignent les différentiations politiques produites par les transformations induites par ladite transition et notamment la précarisation croissante des travailleurs. L'étude minutieuse des attentes des ouvriers, du profil des travailleurs syndiqués et des stratégies dominantes des syndicats remet en cause l'idée que les syndicats représenteraient une "aristocratie ouvrière ». Elle pose ainsi les bases d'une réflexion sur les principaux défis auxquels les syndicats font face à présent, en particulier en termes de représentation des travailleurs.

21 L'article de Bojan Bilić se penche sur le croisement des enjeux de genre et des transformations systémiques de la société serbe postsocialiste. Étudiant la Marche lesbienne organisée à Belgrade en 2015 sur la base du principe de séparatisme (ou nonmixité), il réinscrit la question du séparatisme lesbien dans son histoire régionale, yougoslave et globale. Ce faisant, il rappelle les prémices et les fondements du séparatisme américain des années 1970, ainsi que les débats entre féministes yougoslaves et féministes de l'Ouest qui ont marqué la Conférence « Camarade femme " (Drug-ca žena) organisée en 1978 à Belgrade. L'auteur souligne les difficultés rencontrées lors de cette conférence pour faire valoir l'«effort socialiste d'universaliser (plutôt que de particulariser) l'expérience des femmes en l'appréhendant comme un problème social d'intérêt général ». Il montre ensuite comment les activistes peinent à se réapproprier les acquis du féminisme socialiste dans un environnement capitaliste, caractérisé par un retour du patriarcat et une occultation du passé socialiste. La Marche lesbienne a, selon lui, réifié les frontières binaires de genre en intégrant les cadres des politiques de l'identité associés à la pensée néolibérale contemporaine. In fine, l'auteur appelle les mouvements de lutte, comme les chercheurs qui s'y intéressent, à se réapproprier la complexité des débats féministes de l'époque socialiste, en se rappelant de leurs avancées mais aussi des possibles ouverts à cette période.

22 Angeliki Drongiti étudie les luttes antimilitaristes en Grèce depuis le Mouvement pour l'armée, né dans les années 1980, jusqu'aux associations contemporaines. Elle retrace ainsi les évolutions de la confrontation citoyenne avec l'armée, institution majeure du jeu politique grec. Cette dernière est souvent oubliée dans les débats sur la crise économique en dépit du fait que la Grèce occupe le troisième rang mondial en matière de dépenses militaires. L'article montre comment l'évolution de la lutte antimilitariste, initialement portée par les jeunes conscrits, enrôlés dans les casernes pour leur service 
militaire obligatoire, est marquée par l'histoire des clivages politiques structurant la société grecque post-dictature. Le mouvement antimilitariste, hétérogène et subversif, d'envergure nationale dans les années 1980, se fragmente ainsi en une myriade d'associations aux objectifs plus spécifiques et aux modes d'organisation distincts. La démocratisation de l'armée, initiée dans les années 1980 grâce au Mouvement pour l'armée, se heurte également à la criminalisation des luttes et au contexte socioéconomique délétère. En creux de cette étude socio-historique des luttes antimilitaristes se dessine alors une autre histoire du récit dominant de la «transition ». Si la Grèce est considérée comme un cas à part dans les Balkans, elle a aussi intégré ce récit à son histoire nationale au sortir de la dictature des colonels en 1974. Or, l'article montre que trente ans après l'adhésion à l'Union européenne, la place de l'armée reste cruciale et son pouvoir non négligeable dans le jeu politique grec. L'auteure invite ainsi à interroger le mythe libéral pacificateur à l'heure de la militarisation exponentielle de la frontière orientale européenne.

Toutes les contributions de ce dossier thématique poursuivent l'objectif d'une historicisation critique du paradigme de la transition libérale. Elles le font de manière différenciée en termes d'approches théoriques et méthodologiques, d'objets de recherche et de matériaux mobilisés pour proposer des éclairages heuristiques de l'histoire des mobilisations dans les Balkans. De plus, elles montrent comment les héritages des luttes et des systèmes passés se reconfigurent dans le cadre des transformations structurelles, sociales et politiques, comment ces héritages perdurent dans les modalités d'engagement, mais aussi comment ils peinent à être transmis ou réappropriés.

Il était évidemment impossible de rendre compte de l'ensemble des formes d'action collective expérimentées dans la région ces dernières décennies. D'autres mobilisations mériteraient d'être plus visibles et traitées sous l'angle socio-historique. C'est le cas, par exemple, des mouvements de solidarité et de rejets envers les personnes migrantes sur la « route des Balkans $»^{48}$. L'approche socio-historique appliquée à ces mouvements nous semblerait particulièrement pertinente au regard des expériences de l'exil qui ont durablement marqué les sociétés balkaniques. Il en est de même pour les formes d'action collective hyper-localisées et tenant aux espaces du quotidien - le quartier, la rue, l'espace public - qui gagneraient à être mises en perspective avec les expériences d'organisations locales plus anciennes. Enfin, l'étude des mouvements d'orientation réactionnaire, xénophobe ou d'extrême droite bénéficierait aussi largement du prisme socio-historique afin de faire émerger les trajectoires des acteurs, les reconfigurations des réseaux et des idées dans le temps. Ce travail permettrait sans doute de comprendre plus finement le glissement et l'enchevêtrement des clivages idéologiques (droite/gauche, nationalistes/globalistes...) au sujet desquels les espaces périphériques, dont les Balkans, sont riches d'enseignement. Il est à noter que les mobilisations en Roumanie, en Bulgarie ou en Albanie, à savoir des États postsocialistes qui n'ont pas fait partie de la Yougoslavie, n'ont pas été exclues intentionnellement. Rappelons en effet que le travail de recherche, et particulièrement de publication, est souvent contingent de raisons tout autres que scientifiques, liées à des questions d'opportunités et de disponibilités, mais aussi aux aléas du travail éditorial et collectif, sans parler des 
conditions de travail - de plus en plus précaires - qui tendent à contraindre fortement les contenus. Pour l'ensemble de ces raisons, ce numéro se veut une invitation et une ouverture vers un travail d'historicisation critique de l'étude des mobilisations collectives dans les Balkans, que nous aimerions voir prolongé sur d'autres objets, d'autres espaces qui n'ont pu être explorés ici.

\section{NOTES}

1. BIEBER Florian, BRENTIN Dario (dir.), Social Movements in the Balkans: Rebellion and Protest from Maribor to Taksim, Londres, Routledge, 2018.

2. Vision façonnée dans la longue durée, voir TODORova Maria, Imaginaire des Balkans, Éditions de l'EHESS, 2011 [1997].

3. «Is War About to Break Out in the Balkans? », Foreign Policy, 26 octobre 2015, en ligne : https:// foreignpolicy.com/2015/10/26/war-break-out-balkans-bosnia-republika-srpska-dayton/ (consulté en novembre 2020) ; «Europe. Balkans : une poudrière au cœur de l'Europe ", Courrier international, 16 février 2017, en ligne: https://www.courrierinternational.com/grand-format/ europe-balkans-une-poudriere-au-coeur-de-leurope (consulté en novembre 2020) ; "The next Balkan wars ", Newstatesman, 6 juin 2016, en ligne: https://www.newstatesman.com/world/ 2016/06/next-balkan-wars (consulté en novembre 2020); «La Bosnie-Herzégovine, une poudrière en puissance (OPINION) », La Libre Belgique, 16 février 2018, en ligne: https:// www.lalibre.be/debats/opinions/la-bosnie-herzegovine-une-poudriere-en-puissanceopinion-5a85b243cd7057775ddabb84 (consulté en novembre 2020).

4. Le groupisme renvoie à « la tendance à traiter les groupes ethniques, les nations et les races comme des entités substantielles auxquelles des intérêts et une capacité d'action peuvent être prêtés (...) la tendance à réifier de tels groupes, à parler des Serbes, des Croates, des Musulmans et des Albanais en ex-Yougoslavie (...) comme s'ils étaient des groupes intérieurement homogènes et dotés de limites extérieures, voire des acteurs collectifs unitaires partageant des buts communs (...) par là la tendance à représenter le monde social et culturel comme une mosaïque multichromatique de blocs ethniques, raciaux ou culturels monochromatiques » (BRUBAKER Rogers, "Ethnicity without Groups », European Journal of Sociology/Archives européennes de sociologie, vol. 43, $n^{\circ} 2,2002$, p. 163-189).

5. MiHĂILESCU Vintilă, « Ulysse ou le balkanisme heureux », Civilisations, vol. 60, nº 2, 2012, p. 15.

6. Des travaux ont cependant développé de longue date une approche critique de ces dogmes libéraux, voir à ce propos l'ouvrage suivant, qui regroupe des textes à la fois récents et plus anciens : SAMARY Catherine, LePlat Fred (dir.), Decolonial Communism, Democracy \& The Commons, Dagenham, The Merlin Press, Resistance Book \& IIRE, UK, 2019.

7. BUDEN Boris, « Children of Postcommunism », Radical Philosophy, $n^{\circ} 159,2010$, p. 18-25.

8. Pour la défense de cette thèse voir : коткIN Stephen, Uncivil Society: 1989 and the Implosion of the Communist Establishment, New York, Modern Library, 2009. Pour une critique de cette vision, encore dominante, voir le récent ouvrage de MARK James et al. (dir.), 1989: A Global History of Eastern Europe, Cambridge, Cambridge University Press, 2019.

9. Petrović Tanja (dir.), Mirroring Europe. Ideas of Europe and Europeanization in Balkan Societies, Leiden, Brill, 2014. 
10. SAMPSON Steven, "Weak States, Uncivil Societies and Thousands of NGOs: Benevolent Colonialism in the Balkans ", dans Sanimir Resic, Barbara Törnquist-Plewa (dir.), Cultural Boundaries of the Balkans, Lund, Lund University Press, 2002, p. 27-44.

11. ŽIžEK Slavoj, HORVAT Srećko, What Does Europe Want? The Union and its Discontents, Londres, Istrobooks, 2015.

12. Aucun consensus n'a émergé au sein de la gauche régionale et européenne quant aux réponses à apporter. En témoigne le lancement du mouvement Diem25 par l'ancien négociateur européen pour Syriza, Yannis Varoufakis, soutenu par l'un des promoteurs du Forum subversif de Zagreb, le philosophe Srećko Horvat, se distinguant du Parti de la gauche européenne soutenant Tsipras, alors que des membres de la gauche de Syriza se retrouvaient dans des réseaux alternatifs comme Recommons Europe. Les diverses composantes de cette gauche, a fortiori régionales, partageaient cependant une critique des analyses orientalistes de la Grèce comme " cas à part ", ainsi qu'une perception de l'expérience de Syriza comme symbole d'enjeux européens plus vastes, notamment celui de la périphérisation du Sud et de l'Est de l'Europe. Lire, par exemple, CUKIER Alexis, KHALFA Pierre (dir.), Europe, l'expérience grecque. Le débat stratégique, Vulaines-sur-Seine, Éditions du Croquant, 2015.

13. новSBAWм Eric, L'Âge des extrêmes : le court vingtième siècle (1914-1991), Marseille, Agone, 2020 [1994].

14. BOATCA Manuela, «Semi-peripheries in the World-System: Reflecting Eastern European and Latin American Experiences", Journal of World-Systems Research, vol. 12, nº 2, 2006, p. 321-346; MARK James et al. (dir.), op. cit.

15. PULA Besnik, Globalization under and after Socialism: The Evolution of Transnational Capital in Central and Eastern Europe, Stanford, Stanford University Press, 2018.

16. MARK et al. (dir.), op. cit.

17. KIRN Gal, Partisan Ruptures: Self-Management, Market Reform and the Spectre of Socialist Yugoslavia, Londres, Pluto Press, 2019 ; BonfIGLIOLI Chiara, Women and Industry in the Balkans The Rise and Fall of the Yugoslav Textile Sector, Bloomsbury, 2019 ; ARCHER Rory, DUDA IGOR, STUBBS Paul, Social Inequalities and Discontent in Socialist Yugoslavia, Londres, Routledge, 2016 ; MUsić Goran, Serbia's Working Class in Transition, 1988-2013, Belgrade, Rosa Luxemburg Stiftung South East Europe, 2013.

18. KIRN, Partisan Ruptures, op. cit.; sUVIN Darko, Splendour, Misery and Possibilities: An X-Ray of Socialist Yugoslavia, Leiden, Brill, 2016 ; LUTHER Breda, PUSNIK Maruša (dir.), Remembering Utopia: The Culture of Everyday Life in Socialist Yugoslavia, Washington DC, New Academia Publishing, 2010.

19. BAKER Catherine, Race and the Yugoslav Region: Postsocialist, Post-conflict, Postcolonial?, Manchester, Manchester University Press, 2018.

20. Outre les travaux déjà cités, mentionnons les actes du colloque international organisé par le Centre de recherches historiques de Koper publiés par la revue Acta Histriae, vol. 27, n 1, 2019, en ligne: https://zdjp.si/en/acta-histriae-27-2019-1/ (consulté en octobre 2020) ou encore les conférences internationales « Socialism on the Bench » organisées depuis 2013 par le Centre de recherche culturelle et historique sur le socialisme de l'Université Juraj Dobrila de Pula (Croatie). 21. MADELAIN Anne, GEORGI Frank, Igor DUDA, «Écrire l'histoire de la Yougoslavie socialiste. Savoirs et représentations, usages et circulations ", Revue d'études comparatives Est-Ouest, vol. 1, $\mathrm{n}^{\circ}$ 1, 2019, p. 5-23.

22. FAGAN Adam, SIRCAR Indraneel (dir.), Activist Citizenship in Southeast Europe, Londres, Routledge, 2018 ; BIEBER, BRENTIN (dir.), op. cit ; BILIĆ Bojan (dir.), LGBT Activism and Europeanisation in the PostYugoslav Space. On the Rainbow Way to Europe, Londres, Palgrave Macmillan, 2016 ; CLARKE Jennifer, Huliaras Asteris, Sotropoulos Dimitris, Austerity and The Third Sector in Greece Civil Society at the European Frontline, Londres, Routledge, 2015; ARSENIJEVIĆ Damir (dir.), Unbribable BosniaHerzegovina: The Fight for the Commons, Baden-Baden, Nomos Verlag, 2014 ; BIBIĆ Vedrana, MILAT 
Andrea, HoRVAT Srećko, ŠTIKS Igor (dir.), The Balkan Forum: Situations, Struggles, Strategies, Zagreb, Bijeli Val, 2014.

23. Voir le compte rendu de lecture de Fabio GIOMI et Julia NESTCH dans ce numéro.

24. Fillieule Olivier, Accornero Guya (dir.), Social Movement Studies in Europe: The State of the Art, Oxford, Berghahn, 2016.

25. JACOBSSON Kerstin, SAXONBERG Steven (dir.), Beyond NGO-ization: The Developement of Social Movements in Central and Eastern Europe, Londres, Routledge, 2013.

26. Bilić Bojan, "A Concept That Is Everything and Nothing: Why Not to Study (Post-)Yugoslav Anti-War and Pacifist Contention from a Civil Society Perspective », Sociologija, vol. 53, n 3, 2011, p. 297-322.

27. WUnSCH Natasha, EU Enlargement and Civil Society in The Western Balkans: From Mobilisation to Empowerment, Cham, Palgrave Macmillan, 2018 ; Kostovicova Denisa, BoJICIC-DZELILovic Vesna, KeRLINDSAY James (dir.), Civil Society and Transitions in the Western Balkans Societies and the Crisis of Globalization, Londres, Palgrave, 2016 ; Kоsтоvicova Denisa « Civil Society and Reconciliation in the Western Balkans: Great Expectations?», dans Eviola Prifti (dir.), The European Future of the Western Balkans - Thessaloniki@10 (2003-2013), Paris, European Union Institute for Security Studies, 2013, p. 101-109 ; Simić Olivera, Volčı́ Zala (dir.), Transitional Justice and Civil Society in the Balkans, New York, Springer, 2013 ; Kostovicova Denisa, «Civil Society in the Western Balkans: Vehicle for or Obstacle to Transitional Justice?", dans Wolfgang Petritsch, Vedran Dzihic (dir.), Conflict and Memory: Bridging Past and Future in (South East) Europe, Baden-Baden, Nomos, 2010, p. 287-295.

28. Pour une discussion des différentes approches de cette notion, voir : PERRET Virgile, «Les discours sur la société civile en relations internationales : portée et enjeux pour la régulation démocratique de la mondialisation ", Études internationales, vol. 34, n ${ }^{\circ} 3,2003$, p. 381-399. Voir aussi : STUBBS Paul, «Civil Society or Ubleha? Reflections on Flexible Concepts, Meta-NGOs and New Social Energy in the Post-Yugoslav Space», dans Helena Rill et al. (dir.), 20 Pieces of Encouragement for Awakening and Change: Peacebuilding in the Region of the Former Yugoslavia, Belgrade, Centre for Nonviolent Action, 2007, p. 215-228.

29. Une critique plus détaillée, basée sur des ouvrages récents, a été développée ailleurs. Voir : SAJN Sarah, «L'espace post-yougoslave et l'Union européenne: de l'évaluation balkaniste à l'introspection européenne ", Revue française de science politique, vol. 69, nº 4, 2019, p. 699-703.

30. "Given that many of these 'civil society actors' are involved in an intricate tapestry of private-public-government interactions that secure them a relatively easy access to state institutions and diplomatic parties, instead of talking about civil society, it would be better to talk about a parallel society which has perpetuated an accumulation of various sorts of capital, while at the same time marginalising authentic grassroots initiatives » (BILIĆ, « A Concept That Is Everything and Nothing ", art. cité, p. 311).

31. BRUBAKER Rogers, JUnQUA Frédéric "Au-delà de l' 'identité'", Actes de la recherche en sciences sociales. vol. 139, 2001, p. 66-85.

32. MILAN Chiara, Social Mobilization beyond Ethnicity, Londres, Routletge, 2019 ; TOUQUET Heleen, Escaping Ethnopolis: Postethnic Mobilization in Bosnia-Herzegovina, thèse de doctorat à l'Université KU Leuven, 2011.

33. HORVAT Srećko, ŠTIKS Igor, Welcome to the Desert of Post-socialism! Radical Politics after Yugoslavia, Versobooks, 2014 ; VeTTA Theodora, Democracy Struggles. NGOs and the Politics of Aid in Serbia, New York et Oxford, Berghahn Books, 2018.

34. MAтKоVIĆ Aleksandar, IVKOVIĆ Marjan, « Neoliberal Instrumentalism and the Fight against It: The 'We Won't Let Belgrade D(r)own' Movement », East European Politics, vol. 34, n ${ }^{\circ} 1,2018$, p. 27-38 ; Kušıc Katerina, LotTholz Philipp, Manolova Polina, « Dversia's Special Issue in English: Decolonial Theory \& Practice in Southeast Europe », DвЕРСИЯ, 2019, en ligne : http://dversia.net/ 4644/dversia-decolonial-theory-practice-southeast-europe/ (consulté en novembre 2020). 
35. HoRvat Srećko, Š TIKS Igor, «Welcome to the Desert of Transition: Post-socialism, the European Union, and a New Left in the Balkans ", Monthly Review, vol. 63, n 10, 2012, p. 38-48 ; ŠтікS Igor, SтолAкоvic Krunoslav, "Southeastern Europe's New Left », Rosa Luxemburg Stiftung, février 2019, en ligne : https://www.rosalux.rs/en/southeastern-europes-new-left (consulté en novembre 2020).

36. OfFERLÉ Michel, «13. Histoires de protestations », dans Éric Agrikoliansky (dir.), Penser les mouvements sociaux. Conflits sociaux et contestations dans les sociétés contemporaines, Paris, La Découverte, 2010, p. 265-281 (266).

37. NoIRIEL Gérard, Introduction à la socio-histoire, Paris, La Découverte, 2006, p. 6.

38. Ibid, p. 4.

39. CORCufF Philippe, "Analyse politique, histoire et pluralisation des modèles d'historicité. Éléments d'épistémologie réflexive", Revue française de science politique, vol.61, $\mathrm{n}^{\circ} 6,2011$, p. 1123-1143.

40. Buton François, «L'État et ses catégories comme objets d'analyse socio-historique. Principes, modalités et limites de la production étatique des "handicapés sensoriels" au XIX ${ }^{\mathrm{e}}$ siècle ", dans Pascale Laborier, Danny Trom (dir.), Historicités de l'action publique, Paris, PUF, 2003, cité par CORCUFF, « Analyse politique... », art. cité.

41. Suvin, Splendour, Misery and Possibilities, op. cit. Lire aussi LöWY Michael, Walter Benjamin: Avertissement d'incendie, une lecture des thèses « Sur le concept d'histoire », Paris, PUF, 2001.

42. Verhaeghe Sidonie, Hayat Samuel, Cossart Paula, «Penser le présent, étudier le passé ", Revue française de science politique, vol. 69, $\mathrm{n}^{\circ}$ 2, 2019, p. 327-329 (327).

43. Thомpson Edward P., La formation de la classe ouvrière anglaise, Paris, Gallimard/Seuil, 1988 [1963].

44. TILLY Charles, CASTAÑEDA Ernesto, Wood Lesley J., Social Movements 1768-2018 Fourth Edition, New York, Routledge, 2020 ; TiLly Charles, Contentious Performances, New York, Columbia University Press, 2008 ; SEWELL JR William H., Logics of History. Social Theory and Social Transformation, Chicago, The University of Chicago Press, 2005 ; Doug MCADAM, Sidney TARRow et Charles TILLY, Dynamics of contention, Cambridge, Cambridge University Press, 2004 ; TARROW Sidney, MCADAM Doug, SEWELL JR William H, TiLly Charles et al. (dir.), Silence and Voice in the Study of Contentious Politics, Cambridge, Cambridge University Press, 2001; TiLly Charles, European Revolutions, 1492-1992, Oxford et Cambridge, Blackwell Publishers, 1996 ; MCADAm Doug, Political Process and the Development of Black Insurgency 1930-1970, Londres et Chicago, The University of Chicago Press, 1982.

45. NoIRIEL Gérard, Une histoire populaire de la France. De la guerre de Cent Ans à nos jours, Marseille, Éditions Agone, 2018 ; NoIRIEL Gérard, Les gilets jaunes à la lumière de l'histoire, Avignon, Éditions de l'Aube/Le Monde, 2019.

46. LARRERE Mathilde, Rage against the machisme, Bordeaux, Éditions du Détour, 2020. Voir aussi le dossier «Écrire l"histoire des révolutions : un engagement ", Cahiers d'histoire, n 144, 2019 et notamment « Être une historienne engagée aujourd'hui », entretien avec Mathilde Larrère réalisé par Jean-Charles Buttier, dans Cahiers d'histoire, op. cit., p. 35-51.

47. OFFERLÉ, op. cit., p. 279.

48. Recommandons à ce propos la lecture de l'article de Sasha Hajzler, «From Solidarity to Politics: Transformative Action along the Balkan Migration Route» sur le site https:// www.criticatac.ro/lefteast/from-solidarity-to-politics-transformative-action-along-the-balkanmigration-route/ (consulté le 20 novembre 2020). 
INDEX

Index géographique : Balkans

\section{AUTEURS}

\section{SARAH SAJN}

Doctorante à Sciences Po Aix-en-Provence

sarahsajn[at]hotmail.com

\section{CATHERINE SAMARY}

Économiste, chercheure associée à l'International Institute for Research and Education (IIRE), Amsterdam

amarycatherine[at]yahoo.fr 